PAPER

\title{
Prospective validation of a proposal for diagnosis and management of patients attending the emergency department for mild head injury
}

\author{
A Fabbri, F Servadei, G Marchesini, A M Morselli-Labate, M Dente, T lervese, M Spada, A Vandelli
}

J Neurol Neurosurg Psychiatry 2004;75:410-416

See end of article for authors' affiliations

.......................

Correspondence to: Dr A Fabbri, Dipartimento Emergenza-Urgenza Accettazione, Ospedale GB Morgagni, Azienda USL di Forli, i P.le Solieri-I-47100 Forli, Italy; andfabbri@libero.it

Received in revised form 4 April 2003

Accepted 4 July 2003

Background: In mild head injury, predictors to select patients for computed tomography (CT) and/or to plan proper management are needed. The strength of evidence of published recommendations is insufficient for current use. We assessed the diagnostic accuracy and the clinical validity of the proposal of the Neurotraumatology Committee of the World Federation of Neurosurgical Societies on mild head injury from an emergency department perspective.

Methods: In a three year period, 5578 adolescent and adult subjects were prospectively recruited and managed according to the proposed protocol. Outcome measures were: (a) any post-traumatic lesion; (b) need for neurosurgical intervention; (c) unfavourable outcome (death, permanent vegetative state or severe disability) after six months. The predictive value of a model based on five variables (Glasgow coma score, clinical findings, risk factors, neurological deficits, and skull fracture) was tested by logistic regression analysis.

Findings: At first CT evaluation 327 patients (5.9\%) had intracranial post-traumatic lesions. In 16 cases (0.3\%) previously undiagnosed lesions were detected after re-evaluation within seven days. Neurosurgical intervention was needed in 71 patients $(1.3 \%$ ) and an unfavourable outcome occurred in 39 cases $(0.7 \%)$. The area under the ROC curve of the variables in predicting post-traumatic lesions was 0.906 (0.009) (sensitivity $70.0 \%$, specificity $94.1 \%$ at best cut off), neurosurgical intervention was $0.926(0.016)$ (sensitivity $81.7 \%$, specificity $94.1 \%$ ), and unfavourable outcome was $0.953(0.014)$ (sensitivity $88.1 \%$, specificity $95.1 \%$ ).

Interpretation: The variables prove highly accurate in the prediction of clinically meaningful outcomes, when applied to a consecutive set of patients with mild head injury in the clinical setting of a 1 st level emergency department.

$\mathrm{T}$ e assessment of benefits and hazards in the treatment of patients with mild head injury is of paramount importance for public health. Approximately 200-250 cases per 100000 inhabitants per year are admitted for mild head injury in our region and other European areas. ${ }^{1}$ Hundreds of thousands of patients might benefit from simple and widely applicable guidelines to reduce the risk of death or permanent disability by just a few per cent. ${ }^{2}$

Most patients with mild head injury are currently discharged from the emergency department (ED) without sequelae after a brief observation period. Only a few patients deteriorate within a few days and may require neurosurgical evacuation for an undiagnosed intracranial haematoma. ${ }^{34}$ Early computed tomography (CT) has been extensively applied to these patients, ${ }^{5}$ but its systematic use is not recommended, since only $3-13 \%$ of patients with a Glasgow coma scale (GCS) score of 15 will have a positive CT scan. ${ }^{6} \mathrm{~A}$ systematic use of CT involves high costs and legal problems for inappropriate radiological imaging. ${ }^{7}$ The most reliable approach remains the history, physical examination, simple tests, and a selective use of technology to reach maximum accuracy.

Several studies have been published to identify predictors that would clearly indicate which patients should have CT, and/or prolonged observation period for proper discharge planning. The strength of evidence of current recommendations is not enough for routine clinical use. ${ }^{6}$ In a recent survey more than half of emergency physicians claimed that any clinical decision rule for mild head trauma must have a sensitivity of $100 \%,{ }^{8}$ but budget constraints and technology availability must also be considered.

The Neurotraumatology Committee of the World Federation of Neurosurgical Societies (NCWFNS) has recently proposed a new classification and new clinical guidelines. Minor, mild, or trivial head injury are now defined as "mild" head injury, and a proposal for diagnosis, management, and outcome was put forward after literature review and expert consensus." This proposal allows the diagnostic/therapeutic strategies to be adjusted for locally available resources. In particular, any injury to the head in patients with GCS 14-15 are scored as low risk, medium risk or high risk according to well defined symptoms, signs, and risk factors, and submitted to diagnostic and observation procedures accordingly (table 1 ).

The proposal is still awaiting proper validation, and also the variables selected for classification require further

Abbreviations: AUC, area under the curve; $\mathrm{Cl}$, confidence interval; $\mathrm{CT}$, computed tomography; DSF, depressed skull fracture; ED, emergency department; EDH, epidural haematoma; GCS, Glasgow coma scale; GOS, Glasgow outcome scale; ICH, intracerebral haematoma; IQR, interquartile range; IVH, intraventricular haemorrhage; LR, likelihood ratio; NCWFNS, Neurotraumatology Committee of the World Federation of Neurosurgical Societies; NPV, negative predictive value; $\mathrm{OR}$, odds ratio; PCS, post-concussion syndrome; PPV, positive predictive value; ROC, receiver operating characteristic; SAH, subarachnoid haemorrhage; SD, standard deviation; SDH, subdural haematoma; SR, skull radiography 
Table 1 Criteria proposed by the NCWFS for classification, diagnosis, and treatment of patients with mild head injury

\begin{tabular}{|c|c|c|c|}
\hline & Low risk & Medium risk & High risk \\
\hline GCS & 15 & 15 with clinical findings & $\begin{array}{l}14 \text { or } 15 \text { with neurodeficits or skull } \\
\text { fracture or risk factors with/ } \\
\text { without clinical findings }\end{array}$ \\
\hline Clinical findings & No & $\begin{array}{l}\text { 1. Amnesia } \\
\text { 2. Diffuse headache } \\
\text { 3. Vomiting } \\
\text { 4. Loss of consciousness }\end{array}$ & $\begin{array}{l}\text { 1. Amnesia } \\
\text { 2. Diffuse headache } \\
\text { 3. Vomiting } \\
\text { 4. Loss of consciousness }\end{array}$ \\
\hline Neurodeficits & No & No & Yes \\
\hline Skull fracture & No & No & Yes \\
\hline Risk factors & No & No & $\begin{array}{l}\text { 1. Coagulopathy } \\
\text { 2. Age }>60 \text { years } \\
\text { 3. Previous neurosurgery } \\
\text { 4. Pre-trauma epilepsy } \\
\text { 5. Alcohol and/or drug misuse }\end{array}$ \\
\hline $\begin{array}{l}\text { Imaging } \\
\text { Disposition }\end{array}$ & $\begin{array}{l}\text { No } \\
\text { Home }\end{array}$ & $\begin{array}{l}\text { CT scan or SR } \\
\text { In hospital ( } 3-6 \mathrm{~h} \text { after CT or } 24 \mathrm{~h} \\
\text { after SR), followed by home observation }\end{array}$ & $\begin{array}{l}\text { CT scan } \\
\text { In hospital ( } 24-48 \mathrm{~h}) \text {, followed by } \\
\text { home observation }\end{array}$ \\
\hline
\end{tabular}

CT, computer tomography; GCS, Glasgow coma scale; NCWFS, Neurotraumatology Committee of the World Federation of Neurosurgical Societies; SR, skull radiography.

evaluation in a real setting. We prospectively assessed the diagnostic accuracy and clinical validity of the variables included in the proposal in a population attending a busy lst level ED.

\section{PATIENTS AND METHODS}

\section{Study population and design}

The prospective validation was tested in a three year single centre cohort study, including 6444 consecutive triaged patients, who attended a lst level ED (51 000 patients per year, from a population of 171000 inhabitants) from January 1999 to December 2001. Eligibility criteria were an acute mild head injury, defined by a GCS score of 14 or 15 in patients seen within $24 \mathrm{~h}$ from any trauma to the head in local inhabitants aged $\geqslant 10$ years.

The study profile is given in fig 1. Cases who returned for reassessment after discharge (195 cases) were considered only once.

The patients remaining in the study were managed on the basis of the three different risk categories, ' with minor

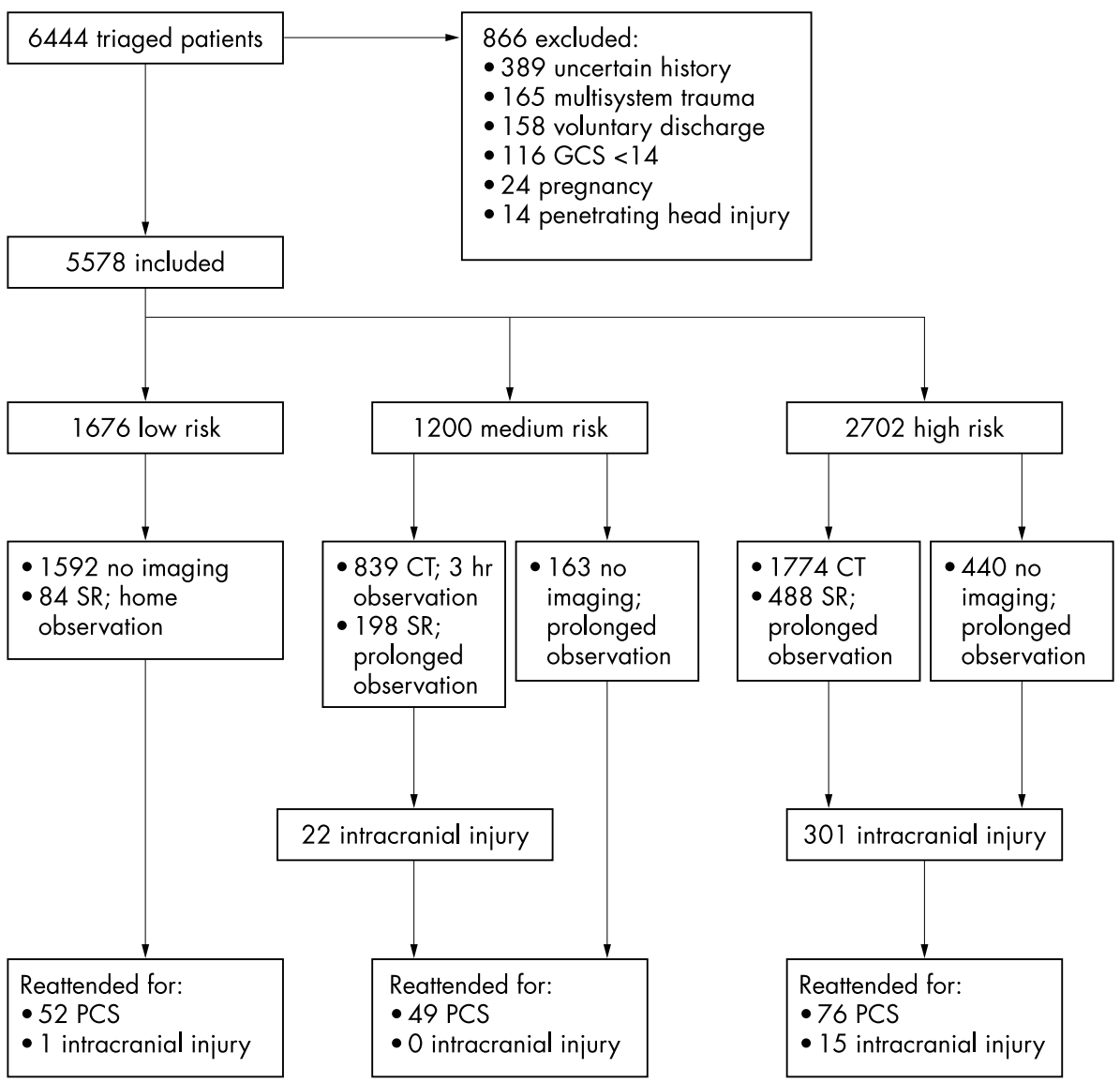

Figure 1 Study profile of patients with different level of risk of complications after mild head injury. 
changes (see below). At discharge, all patients received written recommendations for home observation and complaints that would require referral back to hospital for further evaluation. After re-evaluation and negative CT scan, diagnosis of post-concussive syndrome (PCS) was performed in patients who complained of symptoms like headache, dizziness, nausea, weakness, or abnormal drowsiness. ${ }^{10}$

Observers were instructed to seek symptoms, signs, and any change in the patient's clinical status for seven days. Final outcome was determined approximately six months after injury. A member of the ED staff contacted all patients with a defined post-traumatic lesion by means of a structured telephone interview. In addition, a systematic search of all patients was carried out by checking the death certificates and the medical databases of our local health district. Patients without post-traumatic lesions who were not readmitted for complaints were considered fully recovered.

The protocol was carried out according to the Helsinki Declaration and approved by the senior staff committee of the Azienda Unità Sanitaria Locale of Forlì.

\section{Patient assessment and management}

Patients were classified according to the NCWFNS proposal (table $1^{9}$ ), with minor modifications related to CT availability and budget constraints.

After clinical evaluation, low risk patients were sent home without any radiological imaging. Medium risk patients were submitted to a CT scan and discharged after at least $3 \mathrm{~h}$ from trauma in the case of negative results. Alternatively they had skull radiography (SR), in which case the observation period was prolonged to $24 \mathrm{~h}$. High risk patients were submitted to CT imaging and managed with a first 24 to $48 \mathrm{~h}$ strict observation period, ${ }^{9}$ regardless of CT findings. During the study period, CT was always immediately available during the day, whereas the night service was initially available only on demand. The choice between CT and SR was related to immediate availability of CT, and to the severity of trauma, as judged by the attending physician. To limit the use of CT because of budget constraints, age over 60 years was not considered per se a risk factor.

In our protocol the accepted management options were: (a) monitored in hospital observation; (b) observation in the ED; and (c) home observation under the care of a competent observer, typically a relative or any suitable guardian, after physicians' careful assessment of his/her compliance with instructions.

CT imaging without contrast was performed with a third generation equipment after two or more hours from trauma, with cuts of $10 \mathrm{~mm}$ or less from the foramen magnum to the vertex, and including both soft tissue and bone windows. CT scans showing intracranial post-traumatic lesions were real time reviewed by a neurosurgeon of the nearest neurosurgical unit (Cesena, $17 \mathrm{~km}$ away), consulted via a teleradiology system.

Measurements of blood alcohol levels, as well as toxicological tests for drugs and/or drugs of misuse, were performed at the discretion of the physician in 703 patients $(12.9 \%)$ and in 254 cases $(4.5 \%)$, respectively.

\section{Outcome measures}

Three outcomes were considered: any post-traumatic lesion at CT, need for neurosurgical intervention, and unfavourable outcome.

Post-traumatic lesions, requiring admission to hospital and follow up, included: (a) depressed skull fracture (DSF); (b) intracerebral haematoma (ICH)/brain contusion(s); (c) subarachnoid haemorrhage (SAH); (d) subdural haematoma $(\mathrm{SDH})$; (e) epidural haematoma (EDH); and (f) intraven- tricular haemorrhage (IVH). ${ }^{11}{ }^{12}$ In patients readmitted for complaints a time limit of seven days was set. ${ }^{11}{ }^{12}$

Neurosurgical intervention of haematoma evacuation and/ or skull fracture elevation was only considered in the first seven days after diagnosis. Late events were not considered in the present analysis.

The final outcome at six months was considered unfavourable if the patient died, remained in a vegetative state, or had a persisting severe disability by the Glasgow outcome scale (GOS)..$^{13}$ The follow up GOS was rated by an expert physician unaware of the initial diagnosis, on the basis of the reported response to a structured telephone call.

\section{Analysis}

Mean value, standard deviation (SD), median, range, and frequencies were used to describe data distribution. Hierarchical log-linear model and Kruskal-Wallis test were applied in order to compare baseline characteristics among patients. Univariate and multivariate stepwise logistic regression analysis was used to identify factors related to different outcomes. The odds ratio (OR) and 95\% confidence intervals (95\% CI) were also calculated. A score for risk of posttraumatic lesion was calculated for each patient on the basis of the coefficients computed by the logistic regression derived from variables entering the stepwise procedure. The accuracy of such a risk score was then evaluated by the area under the receiver operating characteristic (ROC) curves. ${ }^{14}$ The best cut off was chosen as the value that maximised the likelihood ratio (LR), computed by the following formula: $\mathrm{LR}=($ probability of true positive + probability of true negative)/(probability of false positive + probability of false negative). ${ }^{15}$ Sensitivity and specificity were considered at best cut off, as well as, the positive predictive value (PPV) and the negative predictive value (NPV).

Non-parametric estimates of the area under the ROC curves (AUC), together with their respective standard errors, were calculated. ${ }^{16}$ Statistical analyses were performed running the SPSS/PC+ statistical package on a personal computer. ${ }^{17}$ Two-tailed p values less than 0.05 were considered statistically significant.

\section{RESULTS}

\section{Study population}

In the final cohort of 5578 adult patients with mild head injury (table 2), high risk cases were less frequently males

Table 2 Clinical characteristics of patients with different level of risk of complications after mild head injury

\begin{tabular}{|c|c|c|c|c|}
\hline & $\begin{array}{l}\text { All cases } \\
(n=5578)\end{array}$ & $\begin{array}{l}\text { Low risk } \\
(n=1676)\end{array}$ & $\begin{array}{l}\text { Medium risk } \\
(\mathrm{n}=1200)\end{array}$ & $\begin{array}{l}\text { High risk } \\
(n=2702)\end{array}$ \\
\hline Sex (males) & $\begin{array}{l}3105 \\
(55.7 \%)\end{array}$ & $\begin{array}{l}990 \\
(59.1 \%)\end{array}$ & $\begin{array}{l}735 \\
(61.2 \%)^{* \star *}\end{array}$ & $\begin{array}{l}1380 \\
(51.1 \%)^{* * *}\end{array}$ \\
\hline $\begin{array}{l}\text { Age (years, } \\
\text { mean (SD)) } \\
\text { Injury type }\end{array}$ & $\begin{array}{l}48.0 \\
(24.2)\end{array}$ & $\begin{array}{l}32.8 \\
(13.0)^{* * *}\end{array}$ & $\begin{array}{l}32.0 \\
(13.2)^{\star * *}\end{array}$ & $\begin{array}{l}64.5 \\
(22.3)^{\star \star *}\end{array}$ \\
\hline Crash & $\begin{array}{l}2819 \\
(50.6 \%)\end{array}$ & $\begin{array}{l}942 \\
(56.2 \%)\end{array}$ & $\begin{array}{l}760 \\
(63.3 \%)\end{array}$ & $\begin{array}{l}1117 \\
(41.3 \%)\end{array}$ \\
\hline Occupational & $\begin{array}{l}\mid 406 \\
(7.3 \%)\end{array}$ & $\begin{array}{l}219 \\
(13.1 \%)^{\text {*** }}\end{array}$ & $\begin{array}{l}113 \\
(9.4 \%)\end{array}$ & $\begin{array}{l}74 \\
(2.7 \%)^{* \star *}\end{array}$ \\
\hline Accidental & $\begin{array}{l}2040 \\
(36.6 \%)\end{array}$ & $\begin{array}{l}382 \\
(22.8 \%)^{* * *}\end{array}$ & $\begin{array}{l}244 \\
(20.4 \%)^{* * *}\end{array}$ & $\begin{array}{l}1414 \\
(52.3 \%)^{* * *}\end{array}$ \\
\hline Assault & 191 (3.4\%) & $86(5.1 \%)^{*}$ & $42(3.5 \%)$ & $63(2.3 \%)$ \\
\hline Sport & $84(2.0 \%)$ & $42(2.5 \%)^{* \star *}$ & $32(2.7 \%)^{* \star *}$ & $10(0.4 \%)^{* \star *}$ \\
\hline Other & $38(0.7 \%)$ & $5(0.3 \%)^{* *}$ & $9(0.8 \%)$ & $24(0.9 \%)^{* \star \star}$ \\
\hline $\begin{array}{l}\text { Injury to } \\
\text { admission time } \\
\text { (min, median } \\
\text { (IQR)) }\end{array}$ & $\begin{array}{l}60 \\
(40-110)\end{array}$ & $\begin{array}{l}60 \\
(40-120)\end{array}$ & $\begin{array}{l}60 \\
(40-120)\end{array}$ & $\begin{array}{l}60 \\
(40-100)\end{array}$ \\
\hline
\end{tabular}

Significantly different from all cases: ${ }^{*} p<0.05 ;{ }^{* *} p<0.01 ;{ }^{* * *} p<0.001$. $I Q R$ : interquartile range. 
Table 3 Clinical variables included in the proposal in patients with different level of risk of complications after mild head injury

\begin{tabular}{llll}
\hline & $\begin{array}{l}\text { Low risk } \\
\text { (n= 1676) }\end{array}$ & $\begin{array}{l}\text { Medium risk } \\
(\mathbf{n = 1 2 0 0 )}\end{array}$ & $\begin{array}{l}\text { High risk } \\
(\mathbf{n = 2 7 0 2 )}\end{array}$ \\
\hline $\begin{array}{l}\text { Glasgow coma score } 1676(100 \%) \\
15\end{array}$ & $1200(100 \%)$ & $2393(88.6 \%)$ \\
Clinical findings & - & $1200(100 \%)$ & $1756(65.0 \%)$ \\
Amnesia & - & $984(82.0 \%)$ & $1581(58.5 \%)$ \\
LOC & - & $320(26.7 \%)$ & $793(29.3 \%)$ \\
Headache & - & $201(16.8 \%)$ & $157(5.8 \%)$ \\
Vomiting & - & $27(2.3 \%)$ & $80(3.0 \%)$ \\
Risk factors & - & - & $2473(91.5 \%)$ \\
Coagulopathy & - & - & $140(5.2 \%)$ \\
Alcohol/drugs & - & - & $506(18.7 \%)$ \\
Previous & - & - & $101(3.7 \%)$ \\
neurosurgery & & - & $138(5.1 \%)$ \\
Pre-trauma epilepsy) & - & - & $1933(71.5 \%)$ \\
Age over 60 years & - & - & $452(16.7 \%)$ \\
Neurological deficits & - & - & $184(6.8 \%)$ \\
Skull fracture & - & & \\
\hline &
\end{tabular}

$(51.1 \%)$, and were older. Median injury to admission time was $60 \mathrm{~min}$ (interquartile range (IQR) 40-110 min), without significant differences among the three risk groups.

Of the medium risk patients, $82 \%$ had amnesia (table 3 ), whereas $91.5 \%$ of high risk cases had one or more risk factors, in particular age over 60 years. Of the 2702 high risk patients, $705(26.1 \%)$ were classified as high risk based only on age.

\section{Diagnostic procedures and management}

The large proportion of low risk patients (95\%) were discharged without any radiological imaging after a brief observation period (median $79 \mathrm{~min}$; IQR, 34-135 min). A large proportion of medium risk patients were submitted to $\mathrm{CT}$, the rest having SR or no radiological imaging.

Only $65.7 \%$ of high risk patients (NCWFNS criteria ${ }^{9}$ ) were submitted to CT scan. This was due to non-systematic consideration of age as a risk factor. Indeed, only 74 of 705 patients aged over 60 had a CT scan (10.5\%). When these patients were excluded from the analysis, 1700 of 1997 cases of high risk patients were submitted to CT scan (85.1\%).

High risk patients were admitted for stricter observation in $38.2 \%$ of cases, the remaining being discharged after an ED observation period (median 114 min: IQR 63-189).

\section{Post-traumatic lesions}

A total of 451 injuries were detected in 327 patients (5.9\%) at first evaluation. A single lesion was present in 224 (68.5\%) of these patients. In addition, 17 previously undiagnosed injuries were detected after re-evaluation in 16 patients (table 4).

Fifty two low risk patients $(3.1 \%)$ reattended the ED after a median of $34 \mathrm{~h}$ (IQR 24-48) for PCS. Only one patient, who reattended after $48 \mathrm{~h}$ for diffuse headache, had an undiagnosed ICH, and he fully recovered at six months without neurosurgical evacuation.

Only 26 medium risk patients $(2.2 \%)$ had post-traumatic lesions at first evaluation or during the first observation period. In patients who reattended the ED (after a median of 24 h, IQR 22-72), only 49 (4.1\%) had PCS.

Of the high risk patients, 301 (5.4\%) had 437 posttraumatic lesions at first evaluation or during the first observation period. Among the 91 who reattended for complaints after a median of $33 \mathrm{~h}$ (IQR 24-60), eight ( $\mathrm{SDH}$, seven cases; SAH, one case) had a negative CT at first evaluation, five ( $\mathrm{SDH}$, three cases; $\mathrm{ICH}$, one case; $\mathrm{EDH}$ one
Table 4 Total post traumatic lesions diagnosed and undiagnosed at first evaluation in patients at different level of risk after mild head injury

\begin{tabular}{|c|c|c|c|}
\hline & $\begin{array}{l}\text { Low risk } \\
(n=1676)\end{array}$ & $\begin{array}{l}\text { Medium risk } \\
(n=1200)\end{array}$ & $\begin{array}{l}\text { High risk } \\
(n=2702)\end{array}$ \\
\hline $\begin{array}{l}\text { Post-traumatic lesions } \\
\text { at first evaluation }\end{array}$ & - & $30(2.5 \%)$ & $421(15.6 \%)$ \\
\hline Depressed skull fracture & - & - & $7(0.3 \%)$ \\
\hline Intracerebral haematoma & - & $17(1.4 \%)$ & $203(7.5 \%)$ \\
\hline Subarachnoid haemorrhage & - & $2(0.2 \%)$ & $66(2.4 \%)$ \\
\hline Subdural haematoma & - & $6(0.5 \%)$ & $108(4.0 \%)$ \\
\hline Epidural haematoma & - & $4(0.3 \%)$ & $27(1.0 \%)$ \\
\hline Intraventricular haemorrhage & - & $1(0.08 \%)$ & $10(0.4 \%)$ \\
\hline $\begin{array}{l}\text { Undiagnosed post- } \\
\text { traumatic lesions }\end{array}$ & $1(0.06 \%)$ & - & $16(0.6 \%)$ \\
\hline Depressed skull fracture & - & - & - \\
\hline Intracerebral haematoma & $1(0.06 \%)$ & & $3(0.1 \%)$ \\
\hline Subarachnoid haemorrhage & - & - & $1(0.04 \%)$ \\
\hline Subdural haematoma & - & - & $10(0.4 \%)$ \\
\hline Epidural haematoma & - & - & $2(0.07 \%)$ \\
\hline Intraventricular haemorrhage & - & - & - \\
\hline
\end{tabular}

case) had a negative SR, and no radiological imaging had been performed in the remaining two cases (EDH, one case; ICH, one case) (table 4).

\section{Neurosurgical intervention}

Neurosurgical intervention was performed in 71 of 5578 patients (1.3\%); none had been classified in the low risk group, five were in the medium risk $(0.4 \%)$, and 66 in the high risk group (2.4\%). Sixty eight patients (63 in the high risk group) were submitted to neurosurgical intervention within seven days. The diagnosis was ICH (4l cases), SDH (25 cases), EDH (13 cases), DSF (4 cases), alone or in combination. In addition, SAH was diagnosed in 17 cases, IVH in two cases. Previously undiagnosed post-traumatic lesions were submitted to neurosurgical intervention only in three high risk patients $(0.1 \%)$ (EDH plus ICH: one case, after 8 h; SDH: two cases after seven days, respectively. A CT scan had not been performed at first evaluation in only one of these patients (undiagnosed EDH plus ICH). He belonged to the high risk group only because of $>60$ years. The remaining two patients had a negative CT at first evaluation.

\section{Unfavourable outcome}

A complete follow up was obtained in 302 of 343 cases with post-traumatic lesions $(88.0 \%)$. Of the remaining 41 cases, six patients died because of complications unrelated to the head trauma, seven were lost to follow up, and in 28 Glasgow Outcome Scale (GOS) was unreliable, due to either a previous disability (24 cases) or a disability unrelated to head injury (four cases).

The outcome was unfavourable in 39 cases $(0.7 \%)$. All patients were high risk; $31(79.5 \%)$ of these died during the hospitalisation for post-traumatic lesions diagnosed at first evaluation. Of these, $21(67.7 \%)$ were older than 75 years. Only three patients (aged 95, 92, and 88 years, respectively) with undiagnosed SDH died following readmission, and no neurosurgical intervention was performed. Among disabilities, one patient remained in a permanent vegetative state and seven cases were severely disabled. The large majority of cases with post-traumatic lesions (263 cases, $87.1 \%)$ had a favourable outcome at six months; only 21 cases had a moderate disability, and 242 fully recovered.

\section{Outcome prediction}

All the variables of the proposal (GCS, clinical findings, risk factors, neurological deficits, and skull fracture) were 


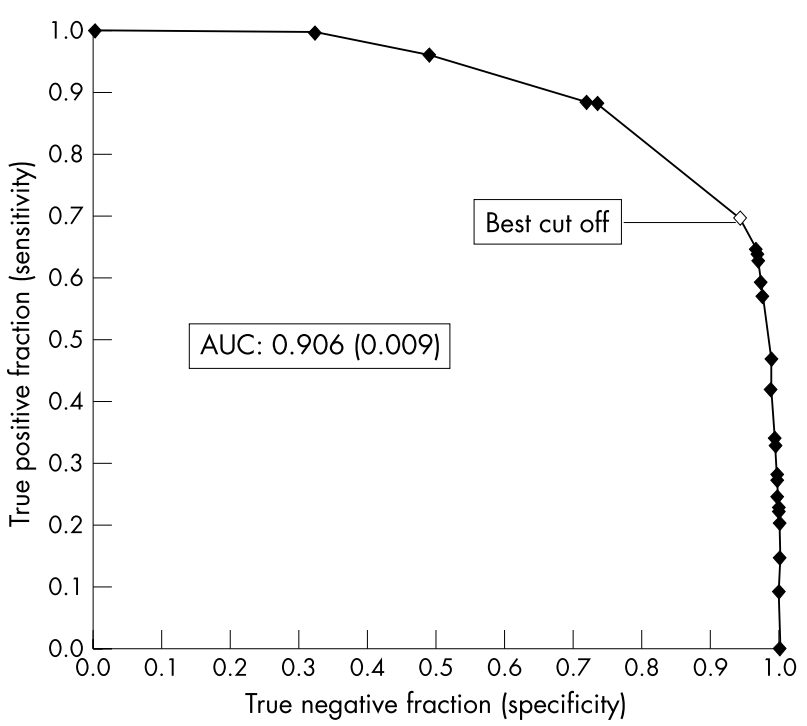

Figure 2 Non-parametric receiver operating characteristic (ROC) plots of the risk score obtained by logistic regression evaluating the procedure (skull fracture, Glasgow coma score (GCS), clinical findings, risk factors, neurological deficits) in identifying patients with post-traumatic lesion after mild head injury. The optimal operating point (best cut off, 2.252.46 ) of the ROC curve is also reported. AUC, area under the curve.

significantly associated with only the presence of posttraumatic lesions at univariate analysis, and were confirmed as independent factors at multivariate stepwise logistic regression (table 5). The ROC curve of the risk score for acute post-traumatic lesions, computed on the basis of the coefficients of the logistic regression (see appendix), is reported in fig 2 . The accuracy in predicting post-traumatic lesions was $0.906(0.09)$ and the best cut off was identified in a score value ranging between 2.25 and 2.46, having a sensitivity of $70.0 \%$ and a specificity of $94.1 \%$ (PPV $43.6 \%$, NPV 98.0\%).

Similarly, the need for neurosurgical intervention was significantly associated to only four variables (table 5), with the exception of risk factors at univariate and neurological deficits at multivariate analysis. The ROC curve had an accuracy of $0.926(0.016)$ and the best cut off resulted a score value ranging between 1.81 and 2.40 , with a sensitivity of $81.7 \%$ and a specificity of $94.1 \%$ (fig 3 ) (PPV $15.1 \%$, NPV $99.7 \%)$.

Finally, the risk for an unfavourable outcome was not associated with clinical findings at multivariate analysis (table 5). The ROC curve had an accuracy of 0.958 (0.014), and the best cut off was a score value ranging between 3.43

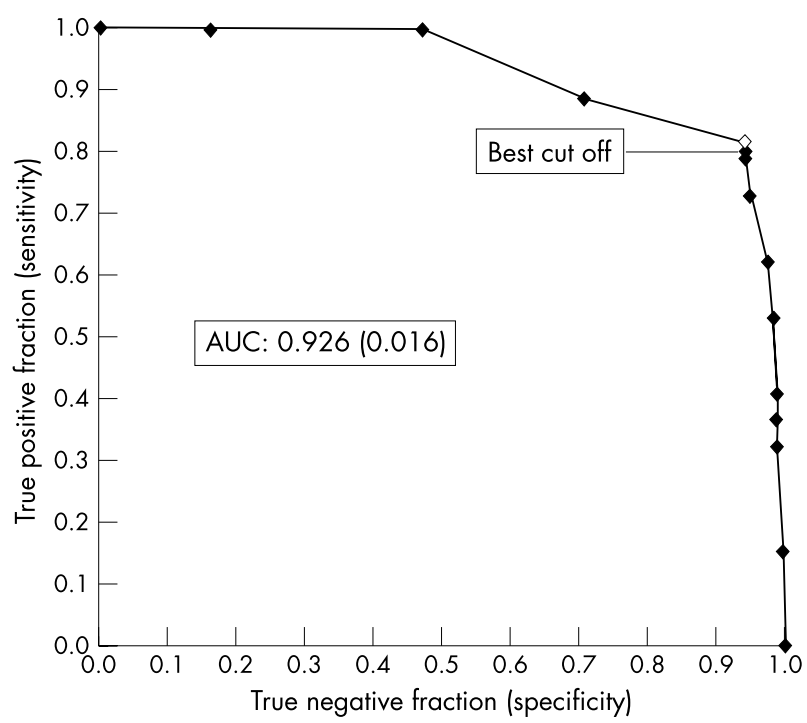

Figure 3 Non-parametric receiver operating characteristic (ROC) plots of the risk score obtained by logistic regression evaluating the procedure (skull fracture, Glasgow coma score, clinical findings, neurological deficits) in identifying patients with neurosurgical intervention after mild head injury. The optimal operating point (best cut off, 1.81-2.40) of the ROC curve is also reported. AUC, area under the curve.

and 3.55 , with a sensitivity of $88.1 \%$ and a specificity of 95.1\% (PPV 11.6\%, NPV 99.5\%) (fig 4).

\section{DISCUSSION}

Our data show that the variables proposed by the NCWFNS are highly accurate for predicting clinically significant outcomes in patients with mild head injury.

A large number of studies have been published to identify which patients should be managed with different options, but they largely vary in patient selection as well as in protocol design, preventing the comparison of different management regimens. ${ }^{2}$

The universal use of CT scan is not cost effective; approximately $90 \%$ of CT scans are negative, and at least $98 \%$ are negative for $\mathrm{EDH}$, the event requiring immediate intervention. ${ }^{11}{ }^{12}$ Also plain SR has been discouraged, because of its very low yield. ${ }^{18}$ The same is true for observation options: in hospital observation cannot be a routine, and home observation by a reliable observer is certainly more cost effective. The NCWFNS proposal put forward a range of practical options deemed acceptable in relation to local resources and budget constraints. ${ }^{9}$ In our protocol, only 2613 of $3902(67.0 \%)$ candidates had a CT scan, the

Table 5 Risk of post-traumatic lesions, neurosurgical intervention, and unfavourable outcome in patients after mild head injury

\begin{tabular}{|c|c|c|c|c|c|c|c|c|c|}
\hline & \multicolumn{3}{|c|}{ Post-traumatic injury } & \multicolumn{3}{|c|}{ Neurosurgical intervention } & \multicolumn{3}{|c|}{ Unfavourable outcome } \\
\hline & $\overline{O R}$ & $95 \% \mathrm{Cl}$ & p Value & $\overline{O R}$ & $95 \% \mathrm{Cl}$ & p Value & $\overline{O R}$ & $95 \% \mathrm{Cl}$ & p Value \\
\hline \multicolumn{10}{|l|}{ Univariate analysis } \\
\hline Skull fracture & 71.00 & 49.67 to 101.48 & $<0.001$ & 48.06 & 29.22 to 79.02 & $<0.001$ & 66.83 & 34.03 to 131.26 & $<0.001$ \\
\hline Glasgow coma score & 37.43 & 28.54 to 49.09 & $<0.001$ & 39.20 & 23.61 to 65.10 & $<0.001$ & 69.06 & 32.44 to 147.02 & $<0.001$ \\
\hline Clinical findings & 8.88 & 6.21 to 12.71 & $<0.001$ & 7.12 & 3.40 to 14.88 & $<0.001$ & 4.15 & 1.83 to 9.42 & $<0.001$ \\
\hline Risk factors & 3.48 & 2.73 to 4.43 & $<0.001$ & 1.22 & 0.77 to 1.96 & 0.395 & 7.10 & 2.97 to 16.97 & $<0.001$ \\
\hline Neurological deficits & 8.47 & 6.62 to 10.84 & $<0.001$ & 6.06 & 3.67 to 10.01 & $<0.001$ & 19.93 & 10.38 to 38.33 & $<0.001$ \\
\hline \multicolumn{10}{|l|}{ Multivariate analysis } \\
\hline Skull fracture & 31.01 & 20.36 to 47.21 & $<0.001$ & 11.06 & 6.05 to 20.23 & $<0.001$ & 10.51 & 4.63 to 23.85 & $<0.001$ \\
\hline Glasgow coma score & 11.74 & 8.42 to 16.37 & $<0.001$ & 13.73 & 7.21 to 26.17 & $<0.001$ & 12.01 & 4.76 to 30.31 & $<0.001$ \\
\hline Clinical findings & 4.31 & 2.81 to 6.61 & $<0.001$ & 2.94 & 1.33 to 6.51 & 0.008 & - & - & 0.951 \\
\hline Risk factors & 2.21 & 1.62 to 3.01 & $<0.001$ & 0.45 & 0.25 to 0.78 & 0.005 & 4.26 & 1.68 to 10.81 & 0.002 \\
\hline Neurological deficits & 1.90 & 1.33 to 2.71 & $<0.001$ & - & - & 0.107 & 2.93 & 1.33 to 6.46 & 0.008 \\
\hline
\end{tabular}




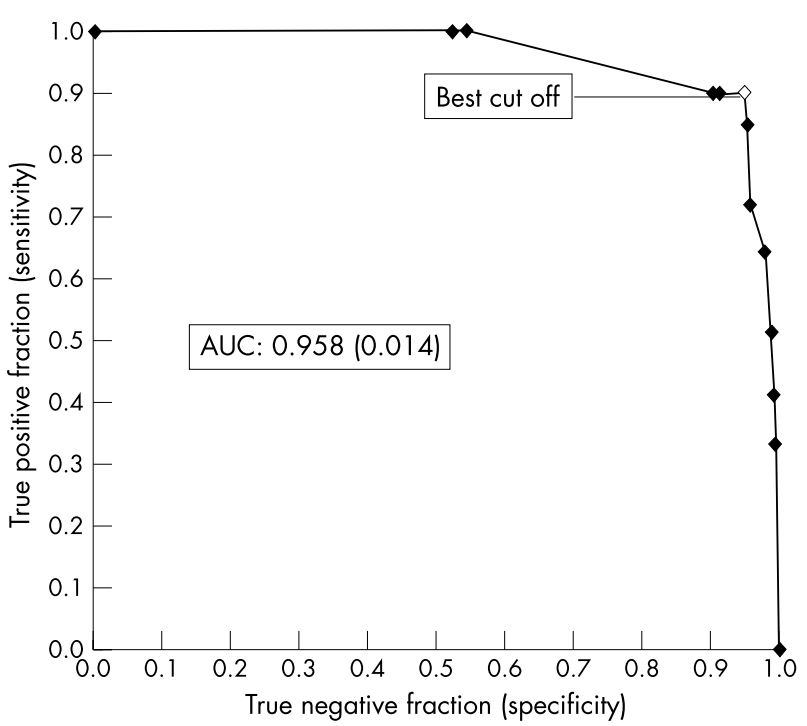

Figure 4 Non-parametric receiver operating characteristic (ROC) plots of the risk score obtained by logistic regression evaluating the procedure (skull fracture, Glasgow coma score, clinical findings, risk factors) in identifying patients with unfavourable outcome (death, permanent vegetative state or severe disability) after mild head injury. The optimal operating point (best cut off, 3.43-3.55) of the ROC curve is also reported. AUC, area under the curve.

remaining having SR followed by a longer observation in ED. The selection was due to immediate availability of resources. The NCWFNS proposal indicates the systematic use of CT for medium and high risk patients, and supports the use of SR only in ED where CT is not available. We extended this concept to the use of SR in any circumstance when CT was not immediately available. A systematic use of CT, also in the presence of available resources, may be limited by organisational problems, and the judgment of the attending physician may be taken into account. In our study, SR was only accepted in patients who were completely intact neurologically and without clinical findings. Whichever strategy was chosen, it is unlikely that patients with post-traumatic lesions were missed. All patients who could not be adequately cared for at home were observed in hospital; patients cared for at home were advised to return in case of new symptoms, and 193 patients $(3.5 \%$ of total cases) reattended for complaints. They were systematically submitted to a CT scan and in 16 of 193 cases (8.3\%) post-traumatic lesions were demonstrated. Finally, patients who did not accept the observation options or who voluntary discharged themselves were excluded. Our ED is the core of a community hospital, serving a well defined population. Also considering that a few injuries were missed, it is conceivable that they did not produce significant symptoms requiring a neurosurgical intervention, or produced an unfavourable outcome. In recent years CT scanning is progressively becoming more available out of hours as primary investigation, but the debate is about how the number of scans can be reduced. This is currently a focus of the National Institute for Clinical Excellence development group in the UK. ${ }^{19}$ The present study provides indirect evidence that the use of CT scanning may be restricted to selected groups.

However, our study was not specifically intended to validate the protocol, but to validate the predictors of outcome. The NCWFNS proposal includes a set of five variables, based on history, physical examination, and radiological imaging that were easy to interpret or to apply real time in a busy ED. ${ }^{9}$ The proposal has limitation as well. Risk factors have an important role, but the NCWFNS did not specify the level of coagulopathy, drug, and/or alcohol consumption for inclusion in the high risk group. It only considered age over 60 years. Increasing age is associated with higher risk, but it is unlikely that there is a specific age threshold above which the risk sharply increases. ${ }^{9}$ This age limit is also a problem. Over $44 \%$ of our patients were over 60 years, and should have been managed as high risk cases. In violation of the NCWFNS proposal, we did not consider age per se a risk factor. Only one of $705(0.1 \%)$ patients classified as high risk according to the proposal reattended with undiagnosed ICH after $48 \mathrm{~h}$. There is probably room for a further simplification.

The validity of any proposal relies on clinically meaningful outcomes, important in terms of patient care and prognosis. ${ }^{11}{ }^{12}$ The likely need for neurosurgical intervention is the most important event to predict in a mildly injured patient. ${ }^{11}{ }^{12}$ The sample size of patients enrolled was a priori considered large enough to test the proposal, but the number of events was lower than expected for each risk group. No low risk cases were submitted to neurosurgical intervention; only five patients were operated on in the medium risk group (expected range of 12 to $36^{\circ}$ ) and 66 in the high risk group (expected, 162 to $270^{\circ}$ ). All cases were inhabitants of the health district referring to our hospital, and it is very unlikely that cases requiring neurosurgical interventions were missed. The number of injuries was as expected; and the number of deaths was not higher than expected. This might reflect a more conservative approach of neurosurgeons, without any detrimental effect on survival. The comparison of different series concerning surgical indications is frequently biased by a number of non-craniotomy procedures (like ICP monitoring), ${ }^{5}$ which are not used in our area in mild head injured patients. Our data confirmed the results obtained by similar studies conducted in the south of Europe. ${ }^{20}$

A highly sensitive proposal would reduce the risk of an unfavourable outcome, ${ }^{12}$ as defined by the dichotomised GOS. ${ }^{132122}$ Telephone referred disability is an additional problem. Structured telephone interviews and questionnaires are likely to underestimate rather than overestimate the results, ${ }^{13}$ but have the advantage of measuring patients' awareness of clinical status.

As outlined by the analysis of ROC plots, the variables proposed by NCWFNS were accurate for the prediction of clinically meaningful outcomes, and specifically for the absence of clinical outcomes, as clearly outlined by PPV and NPV. When the ROC curves were recalculated using the three risk groups defined on the pattern of variables, the proposal maintained a fair to good accuracy. The sensitivity and specificity values for post-traumatic lesions of high versus medium/low risk patients were $92.1 \%$ and $54.4 \%$ (AUC, 0.745 $(0.010))$, those for neurosurgical intervention were $93.0 \%$ and $53.1 \%$ (AUC, $0.736(0.020)$ ), and those for unfavourable outcome were $100 \%$ and $52.3 \%$ (AUC, $0.762(0.022)$ ). These data are in keeping with those reported by Stiell et $a l^{11{ }^{12}}$ for the risk of neurosurgical intervention in a model based on five variables (GCS, suspected or open DSF, any sign of basal skull fracture, vomiting, and age $\geqslant 65$ years), limiting the use of CT to only $32 \%$ of cases. Two additional risk factors (amnesia $>30 \mathrm{~min}$ and dangerous mechanism) guarantee a sensitivity of $98.4 \%$ and a specificity $49.6 \%$ for predicting clinically important brain lesion, with only 54\% CT required. ${ }^{11} 12$ These values were obtained by recursive partitioning analysis, a technique maximising sensitivity on overall accuracy. ROC plots have the advantage to allow a selection of sensitivity/specificity according to specific targets. In our series, increasing the sensitivity in the prediction of post-traumatic lesions to $96.2 \%$ reduces specificity to $48.6 \%$, a figure similar to that reported by Stiell et al. ${ }^{11}{ }^{12}$ Only a 25\% specificity allows a $100 \%$ sensitivity, as reported in a seven 
variable model. ${ }^{23}$ In a low prevalence, high risk disease, the balance between sensitivity and specificity, dependent on number and type of selected variables, is difficult to set.

In conclusion, we believe that the format of the proposal may be adopted by busy physicians in the ED as a reliable, clinically sensible, potentially cost/effective tool for the management of mild head injury patients. The potential for refinement of the proposal, the usefulness of new items, and/ or a different pattern of variables should also be assessed.

\section{ACKNOWLEDGEMENTS}

We are grateful to the emergency medical system personnel for helpful support. We thank the other members of the ED for data collection and participation in the study.

\section{CONTRIBUTORS}

AF conceived the study, wrote the protocol, coordinated the data collection, interpreted the results, and wrote the paper. FS contributed to interpretation of the results and critical review of the paper. GM, contributed to study design, interpretation of the results, and co-wrote the paper. A-MML carried out statistical analyses, interpretation of the results, and co-wrote the paper. MD, TI, and MS were recruited in protocol design, study coordination, data collection, and interpretation of results. AV contributed to study design, study coordination, interpretation of the results, and critical review of the paper. All authors approved the final version of the paper.

\section{Authors' affiliations}

A Fabbri, M Dente, T lervese, M Spada, A Vandelli, Dipartimento Emergenza-Urgenza Accettazione, Ospedale GB Morgagni, Azienda Unità Sanitaria Locale di Forlì, Italy

F Servadei, Divisione di Neurochirurgia per la Traumatologia, Ospedale M. Bufalini, Azienda Unità Sanitaria Locale di Cesena, Italy

G Marchesini, A M Morselli-Labate, Dipartimento di Medicina Interna e Gastroenterologia, Università degli Studi di Bologna, Italy

\section{APPENDIX}

Formula for calculating the scores derived from the stepwise logistic regression:

Score for post-traumatic lesion $=3.434 \times \mathrm{SKU}+2.463 \times$ $(15-$ GCS $)+1.461 \times \mathrm{CLI}+0.79 \times \mathrm{RIS}+0.640 \times \mathrm{NEU}$

Score for neurological intervention $=2.404 \times$ SKU+

$2.620 \times(15-$ GCS $)+1.077 \times$ CLI $-0.807 \times$ RIS

Score for unfavourable outcome $=2.352 \times$ SKU $+2.486 \times$

$(15-\mathrm{GCS})+1.448 \times \mathrm{RIS}+1.076 \times \mathrm{NEU}$

SKU: skull fracture; GCS: Glasgow coma score; CLI: clinical findings; RIS: risk factors; NEU: neurological deficits.

SKU, CLI, RIS and NEU: 0 if absent or 1 if present.

\section{REFERENCES}

1 Servadei F, Verlicchi A, Soldano F, et al. Descriptive epidemiology of head injury in Romagna and Trentino. Comparison between two geographically different Italian regions. Neuroepidemiology 2002;21:297-304.

2 Dickinson K, Bunn F, Wentz R, et al. Size and quality of randomized controlled trials in head injury: review of published studies. BMJ 2000;320:1308-11.

3 Galbraith S. Misdiagnosis and delayed diagnosis in traumatic intracranial haematoma. BMJ 1976;1:1438-49.

4 Marshall LF, Toole BM, Bowers SA. The National Traumatic Coma Data Bank. Part 2: patients who talk and deteriorate: implications for treatment. J Neurosurg 1983;59:285-8.

5 Stein SC, Ross SE. Mild head injury: a plea for routine early CT scanning. J Trauma 1992;33:11-13.

6 Jagoda AS, Kantrill SV, Wears RL, et al. Clinical policy: neuroimaging and decision making in adult mild traumatic brain injury in the acute setting. Ann Emerg Med 2002;40:231-49.

7 Laupacis A, Sekar N, Stiell IG. Clinical prediction rules: a review and suggested modifications of methodological standards. JAMA 1997;277:488-94.

8 Graham ID, Stiell IG, Laupacis A, et al. Emergency physicians' attitude toward the use of clinical decision rules for radiography. Acad Emerg Med 1998;5:134-40.

9 Servadei F, Teasdale G, Merry G, et al. Defining acute mild head injury in adults: a proposal based on prognostic factors, diagnosis, and management. J Neurotrauma 2001;18:657-64.

10 Voss $M$, Knottembelt J, Peden MM. Patients who reattend after head injury: a high risk group. BMJ 1995;311:1395-8.

11 Stiell IG, Wells GA, Vandemheen K, et al. The Canadian CT head rule for patients with minor head injury. Lancet 2001;357:1391-6.

12 Stiell IG, Lesiuk H, Wells GA, et al. The Canadian CT head rule study for patients with minor head injury: rationale, objectives, and methodology for phase I (derivation). Ann Emerg Med 2001;38:160-9.

13 Thornhill S, Teasdale GM, Murray GD, et al. Disability in young people and adults one year after head injury: prospective cohort study. BMJ 2000;320:1631-5

14 Zweig MH, Campbell G. Receiver-operating characteristic (ROC) plots: a fundamental evaluation tool in clinical medicine. Clin Chem 1993;39:561-77.

15 Pezzilli R, Morselli-Labate AM, Miniero R, et al. Simultaneous serum assays of lipase and interleukin- 6 for early diagnosis of acute pancreatitis. Clin Chem 1999;45:1762-7.

16 Hanley JA, MCNeil BJ. The meaning and use of the area under receiver operating characteristic (ROC) curve. Radiology 1982;143:29-36.

17 Norusis MJ, SPSS Inc. SPSS/PC+. Base system and advanced statistics user's guide. Version 5.0. Chicago, Illinois: SPSS Inc., 1992.

18 Bell RS, Loop JW. The utility and futility of radiographic skull examination for trauma. N Engl J Med 1987;316:84-91.

19 National Institute for Clinical Excellence. Head injury in infants, children and adults: triage, assessment, investigation and early management. National Collaborative Centre for Acute Care. http://www.nice.org.uk/pdf/ headinjury_full_version_completed.pdf/

20 Gomez PA, Lobato RD, Ortega JM, et al. Mild head injury: differences in prognosis among patients with a Glasgow Coma Scale score of 13 to 15 and analysis of factors associated with abnormal CT findings. Br J Neurosurg 1996;10:453-60.

21 Teasdale GT, Pettigrew LEL, Wilson JTL, et al. Analysing outcome of treatment of severe head injury: a review and update on advancing use of the Glasgow outcome scale. J Neurotrauma 1998;15:587-97.

22 Ghajar J. Traumatic brain injury. Lancet 2000;356:923-9.

23 Haydel MJ, Preston CA, Mills TJ, et al. Indications for computed tomography in patients with minor head injury. N Engl J Med 2000;343:100-5. 